\title{
Discussion of risks of platinum resources based on a function orientated criticality assessment - shown by cytostatic drugs and automotive catalytic converters
}

\section{Diskussion der Risiken der Ressource Platin auf Basis eines um funktionale Faktoren} erweiterten Kritikalitätsassessments - dargestellt am Beispiel platinhaltiger Zytostatika und Autoabgaskatalysatoren

Andrea Thorenz ${ }^{*}$ and Armin Reller

\begin{abstract}
Purpose: The purpose of the study is the enhancement of criticality assessments for resources in order to address function specific factors like dissipation, recycling, bio-activity and toxicity. The developed methodology is applied to platinum-containing cytostatic drugs and automotive catalytic converters.

Methods: The study is methodically based on an analysis of resource specific factors like exploration rates, reservesto-production ratio and regional distribution of exploration areas as well as on the investigation of product/functional depending factors like recycling rates, dissipation rates, bio-diversity and toxicity. Taking into account that economic and ecological risks may occur at any stage of the supply, consumption and dissipation processes, the whole life cycles of the two analyzed products (cytostatic drugs and automotive catalytic converters) are considered. As an approach to reduce potential economic and ecological risks the study is especially focused on recycling strategies.

Background: In order to get a better understanding of platinum as an essential resource for the development of our society the history and the cultural impact of the term "resource" are introduced.

Results \& Discussion: The availability of platinum is crucial for several products of our modern society. Areas of application are e.g. jewellery, automotive catalytic converters, investments (coins, bars), computers, mobile devices, fertilizers and cytostatic drugs. Economic risks are caused by limited sources and dynamic demand of new application areas like fuel cells and drugs. Platinum-containing drugs are used for the treatment of several kinds of cancer such as testicular, breast, colon and prostate. Currently the pharmaceutical industry requires 6,9 tons per year (3 percent of the total demand of platinum). Due to the improvement of medical standards and the ageing society, especially in developing countries, the demand of platinum-containing drugs will rise significantly. The dissipation of toxic soluble salts and the lack of advanced waste-water treatment and recycling systems is a problem of the
\end{abstract}

*Correspondence: andrea.thorenz@wzu.uni-augsburg.de

Chair of Resource Strategy, Environment Science Center, University of Augsburg, Universitätsstr. 1 a, 86159 Augsburg, Germany

\section{SpringerOpen ${ }^{\circ}$}

(C) 2011 Thorenz and Reller; licensee Springer. This is an open access article distributed under the terms of the Creative Commons Attribution License (http://creativecommons.org/licenses/by/2.0), which permits unrestricted use, distribution, and reproduction in any medium, provided the original work is properly cited. 
usage of platinum-containing drugs. Compared with cytostatic drugs (14.6 kg/a for Germany) the dissipation of platinum particles of mobile catalytic converters is much higher (5-20 tons/a only in North America, Europe and Japan; $184,2 \mathrm{~kg} / \mathrm{a}$ in Germany). On the other hand the emission of platinum nano-particles of automotive catalytic converters seems less critical. Furthermore advanced recycling technologies are already available for them.

Conclusions: The evaluation of resources has to consider resource specific and functional depending factors. In order to reduce economic and ecological risks of the dissipation of platinum salts in cytostatic drugs three principle options can be identified: the development of ecologically compatible and cost-effective substitutes, efficient recycling systems and future-oriented sanitary-systems as well as waste-water treatment facilities, which are able to separate platinum salts. Concerning the dissipation of platinum particles of mobile converters relevant research questions are the identification of adequate substitutes as well as the extension of recycling capacities.

Recommendations and perspectives: The study demonstrates a research gap concerning substitutes for automotive catalytic converters as well as for platinum-containing cytostatic drugs. The development and implementation of recycling concepts, technologies and capacities to reuse platinum as a secondary resource is an essential issue. As a consequence the economic and ecological risks can be reduced by increasing the resource efficiency. Special research has to be done concerning the development of future-oriented sanitary-systems and waste-water treatment facilities in order to separate platinum in waste water. Furthermore, if the future demand of new products like fuel cells is considered, a resource conflict concerning the potential functional applications will be evident. In order to address these challenges the criticality assessment has to be enhanced by ethic and social factors.

Keywords: Criticality Assessment, Dissipation, Recycling, Platinum, Cytostatic Drugs, Automotive Catalytic Converter

\section{Zusammenfassung}

Ziel: Das Ziel der Studie ist eine Erweiterung des Kritikalitätsassessments für Ressourcen um funktionale Faktoren, wie Dissipation, Recycling, Bioaktivität und Toxizität. Die entwickelte Methode wird beispielhaft auf platinhaltige Zytostatika und Autoabgaskatalysatoren angewendet.

Methode: Die Studie basiert methodisch sowohl auf einer Analyse von ressourcenspezifischen Faktoren, wie statische Reichweiten und regionale Verteilung von Abbaugebieten, als auch auf der Erforschung von produkt- bzw. funktionsabhängigen Faktoren, wie Recyclingraten, Dissipationsraten, Bioaktivität und Toxizität. Berücksichtigt man die ökonomischen und ökologischen Risiken in jeder Phase des Wertschöpfungs-, Konsum- und Dissipationsprozesses, so wird deutlich, dass der gesamte Lebenszyklus der zu untersuchenden Produkte (Zytostatika und Autoabgaskatalysatoren) zu betrachten ist. Besonderes Augenmerk wird im Rahmen der vorliegenden Arbeit auf Recyclingstrategien zur Reduktion potentieller ökonomischer und ökologischer Risiken gelegt.

Hintergrund: Zur besseren Einordnung der Bedeutung der Ressource Platin als wichtiger Rohstoff für die Entwicklung unserer Gesellschaft wird zunächst die Kulturgeschichte von Platin bzw. von platinhaltigen Produkten diskutiert.

Ergebnisse und Diskussion: Die Verfügbarkeit von Platin ist ausschlaggebend für eine große Anzahl von Produkten unserer modernen Industriegesellschaft. Zentrale Anwendungsgebiete sind die Schmuckindustrie, Autoabgaskatalysatoren, Investments (wie Münzen und Barren), Computer, Handys, Düngemittel und Zytostatika. Die Verwendung der Ressource Platin wirft gleichermaßen ökonomische, soziale und ökologische Fragestellungen auf. Die ökonomischen Risiken beziehen sich dabei in erster Linie auf die begrenzten Vorkommen, verbunden mit der dynamischen Nachfrage von neuen Anwendungsgebieten, wie Brennstoffzellen und Medikamente. Dies führt zwangsläufig auch zu ethischen Fragestellungen des Einsatzes der knappen Ressource Platin. So werden platinhaltige Medikamente etwa bei der Krebsbehandlung eingesetzt. Derzeit werden in der Pharmaindustrie 6,9 t Platin im Jahr benötigt (3 \% der weltweiten Platinnachfrage). Einhergehend mit der Verbesserung medizinischer Standards und der alternden Bevölkerung, besonders in Entwicklungsländern, wird die Nachfrage nach platinhaltigen Medikamenten signifikant ansteigen. Auf der anderen Seite ist die Ressource Platin unabdingbar für die Entwicklung von Brennstoffzellen und Abgaskatalysatoren. Aus ökologischer Sicht stellt die Dissipation von Platinpartikeln bzw. toxisch wirkenden Salzen (bei der Verwendung von platinhaltigen Medikamenten) ein Problem dar. Diese hängt prinzipiell von der Funktionalität ab. So ist die Dissipation platinhaltiger Salze als Folge der Verwendung von Zytostatika $(14,6$ kg/Jahr in Deutschland) im Vergleich mit der Dissipation von Platinpartikeln der Autoabgaskatalysatoren zwar deutlich geringer (5-20 t/Jahr nur in Nordamerika, Europa und Japan; 184,2 kg/Jahr in Deutschland). Andererseits aber erscheint die Emission von Platinnanopartikeln bei Autoabgaskatalysatoren weniger kritisch. 
Schlussfolgerung: Ein ressourcenorientiertes Kritikalitätsassessment muss sowohl ressourcenspezifische als auch funktionsspezifische Faktoren umfassen. Aufgrund der begrenzten Vorkommen einerseits und der Bedeutung von Platin als Funktionsträger zukunftsorientierter Produkte andererseits entstehen Interessenskonflikte ökonomischer, sozialer, ethischer und ökologischer Art. Zur Lösung dieser Konflikte stehen zwei prinzipielle Optionen zur Verfügung: die Entwicklung von ökologisch kompatiblen und kosteneffizienten Substituten bzw. der Ausbau von effizienten Recyclingsystemen und Entsorgungssystemen.

Empfehlungen und Perspektiven: Die Studie zeigt einen Forschungsbedarf hinsichtlich der Entwicklung von Platinsubstituten für Autoabgaskatalysatoren und platinhaltigen Zytostatika auf. Darüber hinaus ist die Konzeption und Implementierung von Recyclingkonzepten, Technologien und Kapazitäten essentiell. Eine Erhöhung der Ressourceneffizienz führt zur Reduzierung der ökonomischen und ökologischen Risiken. Bei der Entscheidung bezüglich der Verwendung knapper Ressourcen sind Ressourcenkonflikte zu beachten, die bei einer zukünftigen Nachfrage nach neuen Produkten bzw. deren funktionalen Anwendungen entstehen können, wie sich dies beispielsweise bei platinhaltigen Brennstoffzellen zeigt. Zur Bewältigung dieser Herausforderungen ist es notwendig, das Kritikalitätsassessment in Zukunft um ethische und soziale Faktoren zu erweitern.

Schlagworte: Kritikalitätsassessment, Dissipation, Recycling, Platin, Zytostatika, Autoabgaskatalysatoren

\section{Ziel der Studie}

Ziel der Studie ist eine Analyse der Kritikalität der Ressource Platin als Wirkstoff in Zytostatika bzw. als funktionaler Bestandteil von Katalysatoren zur Abgasreinigung in der Automobilindustrie. Dabei stehen neben ressourcenspezifischen Kriterien wie etwa statische Reichweite, geographische Verteilung, insbesondere auch produkt- bzw. funktionsabhängige Kriterien wie das Recyclingpotential und die Dissipation im Vordergrund.

\section{Stand der Kritikalitätsforschung}

Prinzipiell stellt die Analyse und Bewertung der Kritikalität metallischer Rohstoffe ein noch sehr junges und damit heterogenes Forschungsfeld dar. Die bislang entwickelten Methoden zur Bewertung der Kritikalität für einen Kritikalitätsansatz sind nicht eindeutig und zeichnen sich hauptsächlich durch quantitative Ansätze aus. Die amerikanische National Academy of Sciences (NAS) führt in einer Studie ein Kritikalitätsassessment durch, dessen zentrale Komponenten die Bedeutung der Nachfrage, das Substitutionspotential sowie Risikofaktoren bezüglich des Angebots umfassen. Im Einzelnen werden die Verfügbarkeit, politische, regulatorische und soziale Faktoren sowie die Co-Abhängigkeit von anderen Märkten und die Marktmacht einzelner Anbieter in einer Matrix gewichtet und abgebildet. Die größten Gewichtungen liegen dabei auf der Nachfrage (75\%) sowie der Verfügbarkeit (40\%) [1]. In einer Studie der Europäischen Kommission wurde ein Kritikalitätsassessment durchgeführt, das sich ebenfalls auf die geologische und technologische Verfügbarkeit, die Reichweite, die Nachfragerisiken (Konzentrationsniveau, Substitution und Recycling) und die ökonomische Bedeutung der kritischen Metalle bezieht [a]. Die Betrachtung wurde hier allerdings um ökologische Risiken erweitert. Darunter verstehen die
Autoren allgemeine Maßnahmen, die von den Ländern getätigt werden, um die Umwelt zu schützen. Politikorientierte Empfehlungen sollen die Zugangssicherheit und die Materialeffizienz gewährleisten [2].

\section{Methode}

Das methodische Vorgehen der vorliegenden Arbeit basiert auf einer um funktionale Kriterien erweiterten Kritikalitätsanalyse. Dabei wird die gesamte Wertschöpfungskette der relevanten Produkte bzw. deren funktionaler Gruppen hinsichtlich ökonomischer und ökologischer Wirkungen untersucht. Die zur Bewertung benötigten Stoffströme werden anhand einer Stoffstromanalyse des Verteil- und Wertschöpfungsnetzes ermittelt (Abbildung 1). Auf Basis dieses Ansatzes wird die Kritikalität der Ressource Platin als Wirkstoff platinhaltiger Zytostatika bzw. als Autoabgaskatalysator in Abgasreinigungssystemen vergleichend diskutiert.

Die bislang entwickelten Kritikalitätsansätze zeichnen sich im Wesentlichen durch eine Bewertung funktionsunabhängiger Kategorien wie „Regionale Konzentration von Abbaugebieten“, „aktuelle Angebots- und Nachfragemengen“" „statische Reichweiten der zu fördernden Ressource" und "Substitutions- und Recyclingpotential" aus. Dabei werden spezifischere, ökologische Bewertungskategorien wie "Dissipation" und dessen Wirkung auf die „Bioaktivität“ und "Toxizität“ vernachlässigt. Diese hängen von der Art der Verwendung in den jeweiligen Produkten ab. Insofern ist die Kritikalität einer Ressource stets in Bezug auf ihre Funktion (z.B. Wirkstoff von Platin in Zytostatika, katalytische Eigenschaft von Platin in Autoabgaskatalysatoren) in einem oder mehreren Produkten zu sehen. Ein weiteres Defizit liegt in der oftmals singulären Betrachtung einzelner Prozesse der Wertschöpfungskette, wie z.B. der „Förderung“ und 


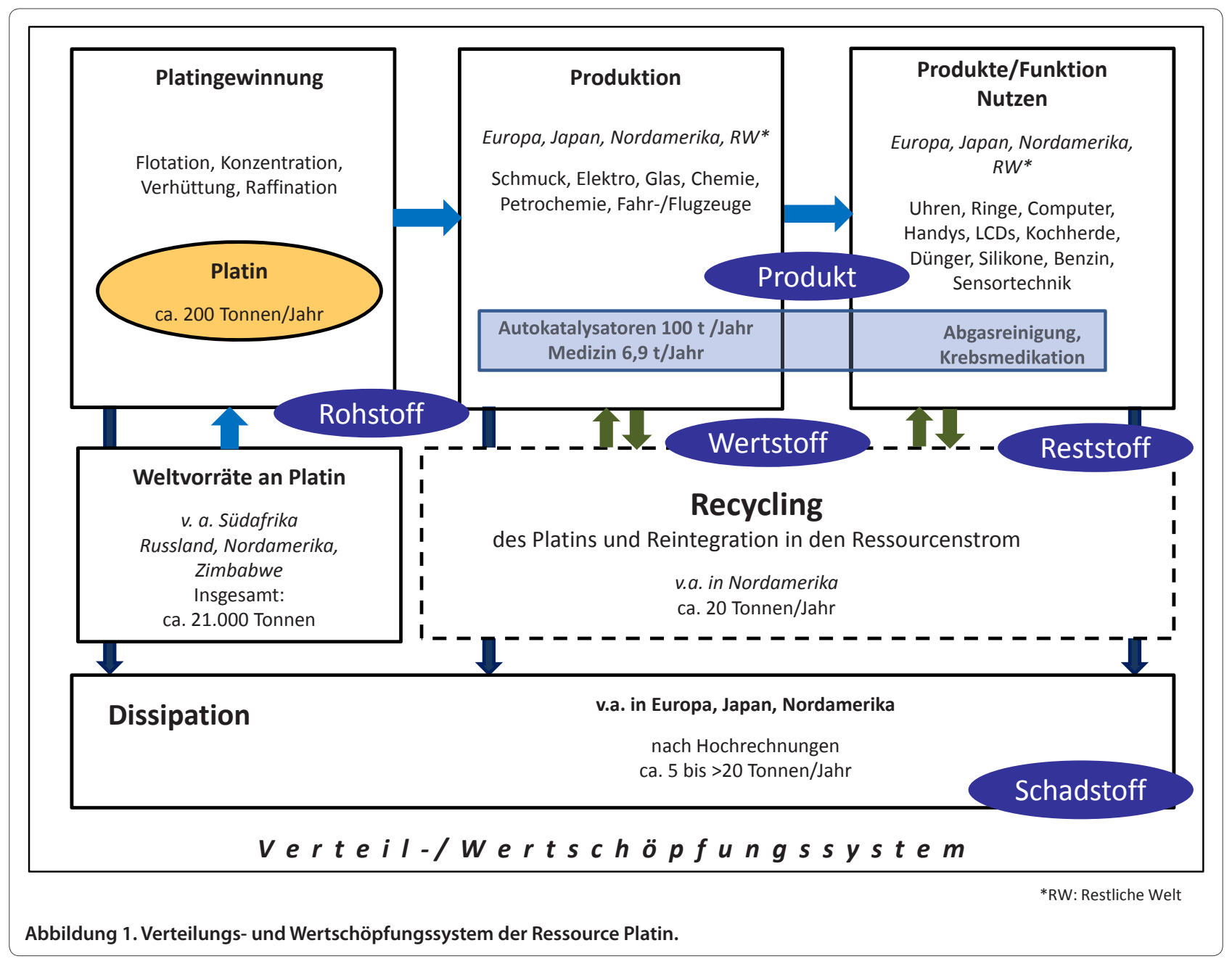

„Produktion“. Im Sinne einer ganzheitlichen Bewertung der zu untersuchenden Produkte oder Funktionsgruppen sind aber alle Prozesse von der Förderung und Gewinnung der Ausgangsressourcen über die eigentliche Herstellung der funktionalen Gruppen bis hin zur Nutzung und Entsorgung zu untersuchen. Dabei sind neben ökonomischen Kriterien gleichermaßen auch ökologische Implikationen zu berücksichtigen, insbesondere für die Förder-, Nutzungs- und Entsorgungsphase.

Basis dieser ganzheitlichen Bewertung ist eine Stoffstromanalyse, die sich von den Quellen (Verteilungssystem) über die Produkte (Produktions- und Nutzungssystem) bis zur Entsorgung (Dissipation und Rückführung) erstreckt. Dabei wird zur Abschätzung ökologischer Risiken ein besonderes Augenmerk auf die Dissipation gelegt. Grund hierfür ist die Tatsache, dass die Dissipation einen unkontrollierten, irreversiblen Eintrag von potentiellen Schadstoffen in die Biosphäre darstellt und zudem infolge von Kreislaufverlusten die Förderung zusätzlicher Primärrohstoffe impliziert. Als
Lösungsansatz zur Reduktion potentieller ökologischer Risiken wird das Potential von Rückführungsprozessen (im Sinne der Umsetzung von Kreislaufwirtschaftssystemen) untersucht.

\section{Hintergrund - Kulturgeschichte des Platins}

Für ein tiefergehendes Verständnis der Bedeutung der Ressource Platin, das als "Weißgold“ seit jeher die Menschen interessiert (im Sinne der funktionalen Nutzung), ist zunächst eine Analyse der Kultur- und Technikgeschichte dieses zweitwertvollsten Edelmetalls (nach Rhodium) erforderlich. Die "Stoffgeschichte“ von Platin, auch als das „neue Metall“ bezeichnet, ist viel älter als man zunächst glaubt. Annahmen gehen davon aus, dass es - wie die Elemente Gold, Iridium, Rhodium und Palladium - vor mehr als vier Milliarden Jahren durch Meteoriteneinschläge in die Erdkruste gelangte. Grund für diese Annahme ist die Tatsache, dass es sich mit Eisen verbindet und deshalb eigentlich nicht in der Erdkruste, dafür aber im eisenreichen Erdkern vorkommen müsste. 
So aber fanden die alten Ägypter und die Inkavölker nicht nur das Gold, sondern auch das silberglänzende Platin in den Flüssen und verarbeiteten es zu Schmuck. Lange Zeit wurde Platin als „wertloser Stoff“ klassifiziert, für den man keine Verwendung fand. Deshalb wurde das Metall, auch wegen seiner grau-weißen Farbe als „Platina“, als „kleines Silber“, bezeichnet. Einzig zur Fälschung des zur damaligen Zeit begehrtesten aller Metalle, des Goldes, schien es brauchbar zu sein. Letztendlich waren es die spanischen Eroberer, die das Platin nach Europa brachten. Die erste europäische Erwähnung stammt von dem Italiener und Humanisten Julius Caesar Scaliger. Er beschreibt ein mysteriöses weißes Metall, das sich allen Schmelzversuchen entzog. Eine ausführlichere Beschreibung der Eigenschaften findet sich erstmals in einem 1748 veröffentlichten Bericht von Antonio de Ulloa [3]. Daraufhin dauerte es noch viele Jahre bis die Verfahren für die Bearbeitung perfektioniert werden konnten. Zunächst wurde Platin im Kunsthandwerk für Schmuckwaren und zur Dekoration von Porzellan sowie zur Herstellung von Labortiegeln und Drahtgeflechten eingesetzt. Später war es, bis zur Substitution durch günstigere Materialien, wichtiger Bestandteil epochaler Entwicklungen des 19. Jahrhunderts, wie etwa dem elektrischen Telegraphen oder der Glühbirne. Seitdem wird es - vorwiegend aufgrund seiner katalytischen Eigenschaften - bei Fahrzeugkatalysatoren eingesetzt. Ein zukünftig besonders interessantes Einsatzgebiet liegt in der Entwicklung von Brennstoffzellen. Dort wird Platin ebenfalls als funktionaler Bestandteil von Katalyseprozessen benötigt. Darüber hinaus kommt es in der Schmuckindustrie, in Laborgeräten, Zahnimplantaten und in der Pharmaindustrie bei der Herstellung von Zytostatika in der Chemotherapie von Krebspatienten zum Einsatz. Neueste Anwendungsgebiete zeigen sich vermehrt in der Automobilindustrie beim Einsatz in Brennstoffzellen.

\section{Ergebnisse}

Die Analyse der ökologischen Risiken des Einsatzes der Ressource Platin erfolgt anhand des in Abbildung 1 beschriebenen Verteilungs- und Wertschöpfungssystems. Bei der Untersuchung des Verteilsystems werden insbesondere die Ergiebigkeit der derzeit bekannten Quellen und deren geographische Verortung analysiert. Im Rahmen der Erörterung der Herstell- und Verwertungsprozesse liegt der Fokus auf der Struktur der Nachfrage platinhaltiger Produkte im Allgemeinen sowie einer Diskussion platinhaltiger Zytostatika und Autoabgaskatalysatoren im Besonderen. Die Risiken platinhaltiger Katalysatoren und Medikamente für die Biosphäre ergeben sich insbesondere aus dem Phänomen der Dissipation. Eine potentielle Option zur Reduktion dieser Risiken liegt in der Schaffung effizienter
Rückführungssysteme. Daher werden im Weiteren der Stand der Rückführungssysteme verglichen sowie deren strukturelle Grenzen diskutiert.

\section{Analyse der Angebotsstruktur der Ressource Platin}

Eine Analyse der Quellen (Verteilungssystem) zeigt, dass es sich bei Platin um ein relativ seltenes Metall handelt, welches zudem nur in wenigen, regional stark konzentrierten Abbaugebieten gefördert wird. So war bis 1820 die einzige bekannte Quelle für Platinmetalle Kolumbien. Erst mit der Entdeckung von Vorkommen im Ural kam Russland (Norilsk) als weiteres Fördergebiet hinzu. Vor ca. 90 Jahren wurden in Südafrika (Bushveld Komplex) die bis heute bedeutendsten Lagerstätten entdeckt. Abbildung 2 zeigt die Aufteilung der Platinvorkommen nach Regionen.

Die derzeit größten Fördergebiete befinden sich in der Region um Johannesburg (Südafrika). Dort werden ca. 77\% der aktuellen Fördermengen abgebaut. Weitere wichtige Förderregionen sind Russland mit 13\%, Nordamerika mit $4 \%$ und Zimbabwe mit $4 \%$. Die jährliche Primärproduktion von Platin liegt bei ca. $200 \mathrm{t}$ und der prognostizierte Ressourcenvorrat bei ca. 21.000 t Platin. Damit ergibt sich eine hypothetische Reichweite von etwa 100 Jahren. Die weltweite Förderung ist auf wenige große Minengesellschaften konzentriert, die über eigene Verhüttungs- und Raffinationsbetriebe verfügen [4].

Charakteristisch für den Abbau und die Raffination von Platin bzw. den Metallen der Platingruppe (Platin, Palladium, Rhodium, Ruthenium, Iridium und Osmium) ist der energieintensive Gewinnungsprozess. Der Abbau von Platin findet im Allgemeinen Untertage statt. Der durchschnittliche Gehalt von Platin in der Erdkruste beträgt nur ca. 1/2000 Prozent. Um eine Feinunze (ca. 30 g) Platin zu erhalten, müssen $7-12$ t erzhaltiges Gestein bewegt werden, d.h. gesprengt, zermahlen und sowohl chemisch als auch mechanisch behandelt werden. Neben der direkten Förderung werden die Metalle der Platingruppe als Nebenprodukt, z.B. bei der Förderung von Nickel, Kupfer, Gold oder Silber gewonnen [5]. Dies stellt insofern ein Problem dar, als dass bei der Einstellung der Förderung dieser Metalle auch die Verfügbarkeit der Kuppelprodukte Platin, Palladium und Rhodium, abnehmen würde. Darüber hinaus bedingt die Förderung von Platin zahlreiche ökologische Probleme, wie den Einsatz von kritischen Chemikalien und die Veränderung des Landschaftsbildes.

\section{Analyse der Nachfragestruktur platinhaltiger Produkte}

Aus ökonomischer Sicht ist neben einer Analyse der derzeit bekannten Fördergebiete insbesondere die Struktur der Nachfrage nach platinhaltigen Produkten bzw. Funktionsgruppen von zentraler Bedeutung. Dabei sind in einem ersten Schritt diejenigen Produkte zu 


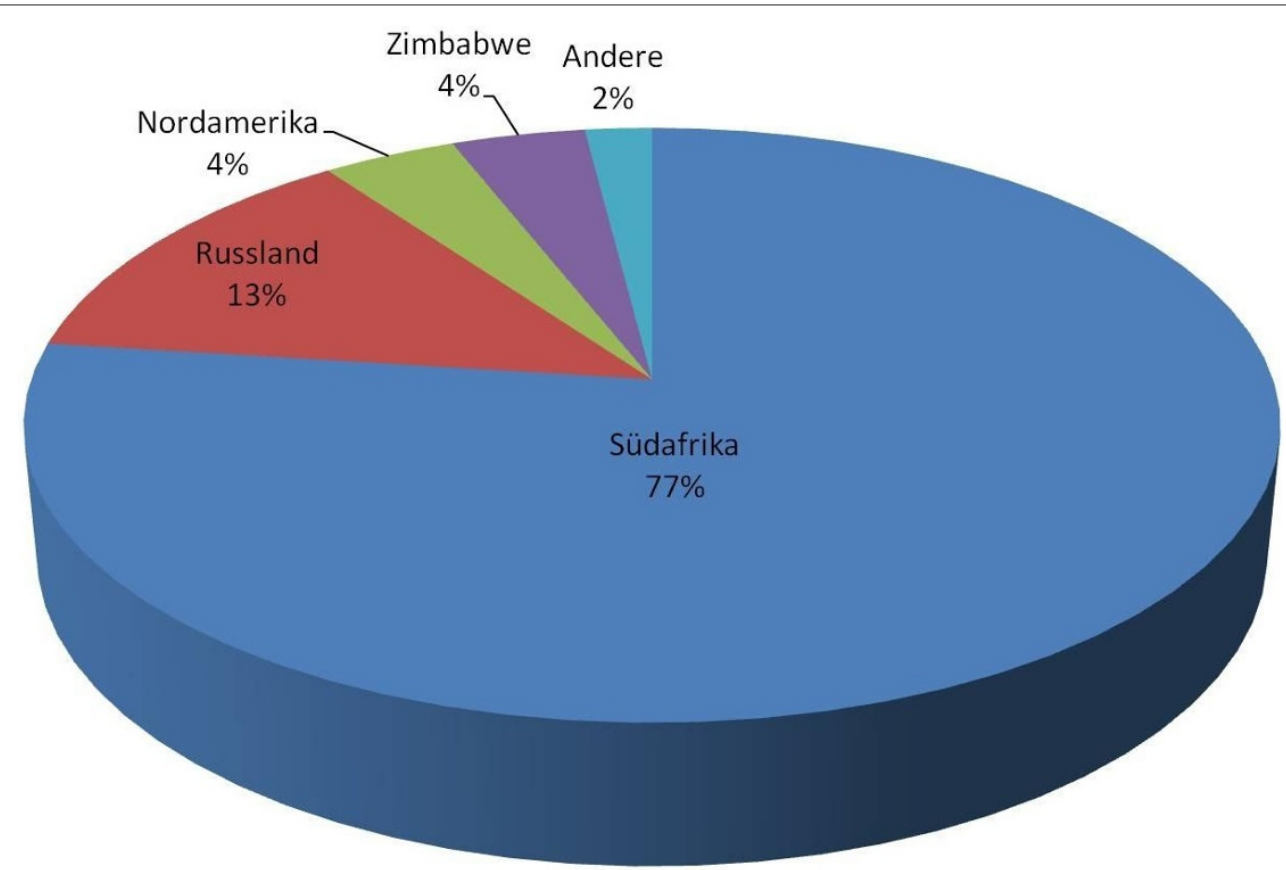

Abbildung 2. Regionale Aufteilung der Platinforderung.

identifizieren, für die Platin ein funktional notwendiger Bestandteil ist. Im Weiteren sind die Bedarfe der entsprechenden Produkte bzw. Branchen zu bestimmen. Darauf aufbauend kann die statische Reichweite einer Ressource als eine der zentralen Kategorien der Kritikalität eines Rohstoffes ermittelt werden.

Entsprechend der mengenmäßigen Bedeutung von Autoabgaskatalysatoren sowie der zunehmenden Bedeutung platinhaltiger Medikamente wird im Weiteren ein spezieller Fokus auf diese funktionalen Verwendungen gelegt.

\section{Klassifikation der Platinnachfrage}

Platin ist wirtschaftlich sehr bedeutend und Funktionsträger in vielen Anwendungen, für die schwer bzw. gar keine Substitutionsmöglichkeiten bestehen [6]. Untersucht man die Nachfragesektoren platinhaltiger Produkte, lassen sich acht Hauptgruppen identifizieren: Schmuck- und Autoindustrie (im Wesentlichen Autoabgaskatalysatoren), Investments (physische Münzen und Barren), Chemiesektor (Einsatz in chemischen Prozessen sowie bei der Herstellung von chemischen Geräten), Glasindustrie, medizinischer bzw. biomedizinischer Sektor (z.B. Verwendung im Dentalbereich, Wirkstoffe für Zytostatika), Elektroindustrie (Computer, Handys und LCDs) und Mineralölveredelung (siehe Abbildung 3). Dabei zeigt sich in der Schmuckund Automobilindustrie die größte Nachfrage.

Die Gesamtnachfrage nach Platin lag im Jahre 2008 weltweit bei $250 \mathrm{t}$ (im Jahre 2009 waren dies aufgrund der
Finanzkrise nur 195 t). Dabei ist zu beachten, dass die Nachfrage eine steigende Tendenz aufweist (z.B. durch das weltweite Wachstum in der Automobilindustrie) und über dem Angebot liegt. Dementsprechend ist der Preis für Metalle der Platingruppe in den letzten Jahren an den Rohstoffbörsen stetig gestiegen (im April 2011 betrug der Preis 1.250 Euro je Feinunze). Eine Möglichkeit die Nachfragelücke zu schließen, liegt in der Umsetzung von Kreislaufwirtschaftssystemen. Derzeit wird das in Autoabgaskatalysatoren, der Schmuckindustrie und in elektronischen Produkten enthaltene Platin mit unterschiedlicher Effektivität recycelt. So konnten im Jahre 2008 bei Autoabgaskatalysatoren 32 t, in der Schmuckindustrie 19,7 t und im elektronischen Sektor 0,13 t weltweit rezycliert werden [4].

\section{Einsatz von Platin in der Automobilindustrie}

Ein bedeutendes Einsatzgebiet von Platin ist dessen Verwendung in Autoabgaskatalysatoren. Die gesamte Abgasreinigungseinheit besteht aus einem Trägermaterial (Monolith) - gefertigt aus Keramik oder Metall - durch dessen Kanäle das Abgas strömt. Diese Strömungskanäle sind mit einer katalytisch aktiven Schicht aus Platingruppenmetallen (Platin, Palladium und Rhodium) beschichtet. Dabei liegt die funktionale Relevanz von Platin im Oxidationsprozess (Rhodium im Reduktionsprozess). Im Autoabgaskatalysator reagieren Kohlenstoffmonoxid sowie nicht verbrannte Kohlenwasserstoffe mit Stickoxiden und Sauerstoff zu ungiftigem Kohlenstoffdioxid sowie Stickstoff und Wasser. So können die 


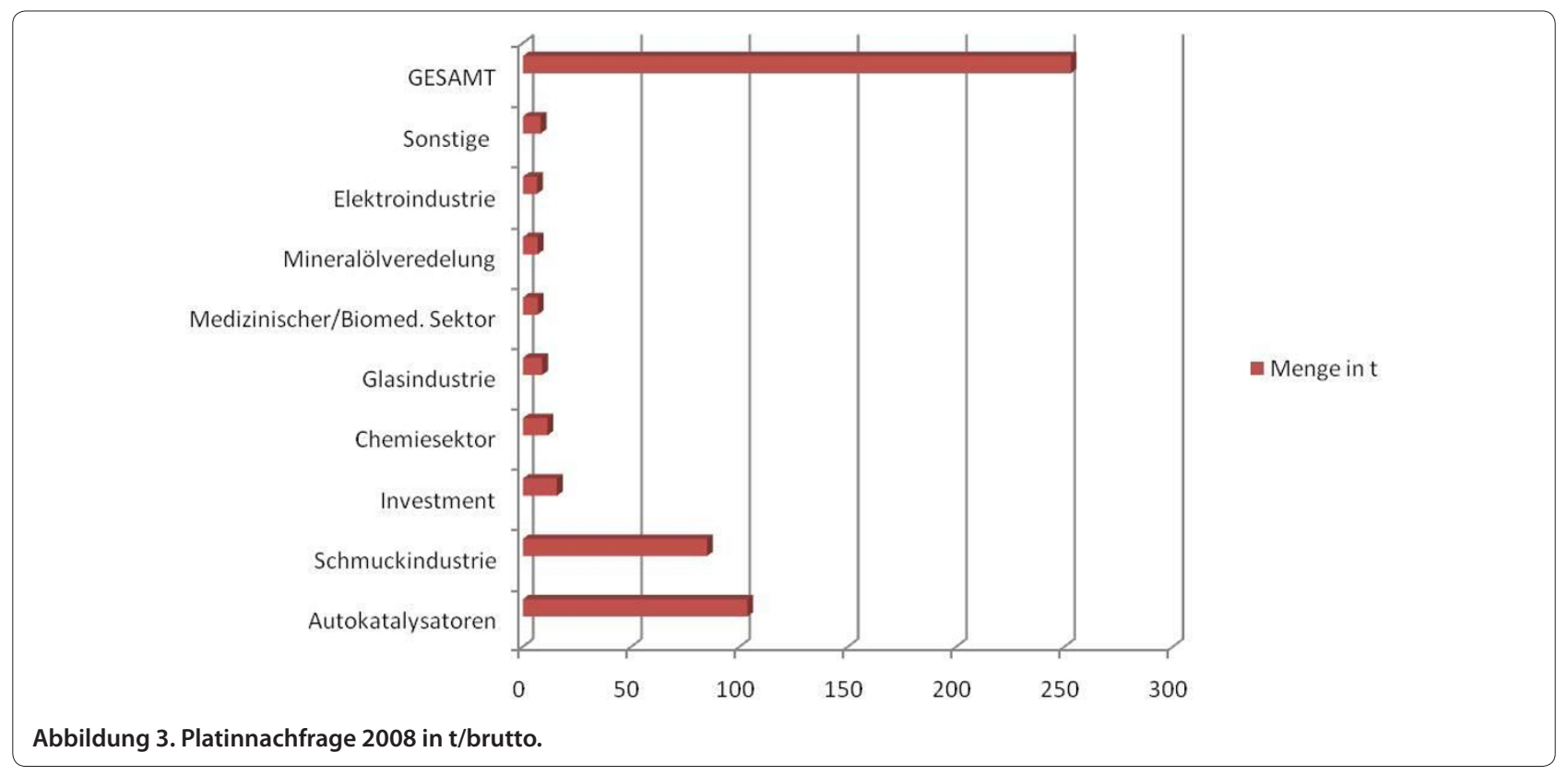

Schadstoffemissionen (Kohlenwasserstoffe, Stickoxide, Kohlenmonoxid) um bis $\mathrm{zu} 95 \%$ verringert werden. Derzeit sind ca. 85\% aller Autos weltweit mit Katalysatoren ausgestattet. Bei Dieselmotoren und DreiWege-Katalysatoren handelt es sich um Oxidationskatalysatoren, bei denen hauptsächlich Platin (beim Dieselmotor mittlerweile auch Palladium) eingesetzt wird. Die eingesetzte Platinmenge beträgt 1-3 g pro Katalysator $[5,7,8]$.

Ein zukünftig wichtiges Einsatzgebiet von Platin, mit der Zielsetzung der $\mathrm{CO}_{2}$-Reduzierung, sind Brennstoffzellen in der Automobilindustrie. Diese stellen eine Alternative zu batteriegetriebenen Antrieben dar, die durch die Verfügbarkeit leistungsfähiger Batteriesysteme bzw. den dafür benötigten Ressourcen wie etwa Lithium begrenzt sind. Das Platin in den Brennstoffzellen hat ebenfalls eine katalytische Wirkung. Die in der Knallgasreaktion freiwerdende Energie wird elektrochemisch kontinuierlich nutzbar gemacht. Problematisch beim Einsatz der mit Brennstoffzellen betriebenen Automobile, die 2015 auf den Markt kommen sollen, ist somit die Verfügbarkeit von Platin. Eine Brennstoffzelle benötigt nach derzeitigem Stand der Forschung - ca. 10 g Platin und kann derzeit nicht substituiert werden $[2,6,9]$.

\section{Einsatz von Platin im medizinischen Sektor}

Ein besonderes innovatives Einsatzgebiet von Platin ist der medizinische Sektor. Prinzipiell lässt sich feststellen, dass metallhaltigen Arzneimitteln in der Medizin eine steigende Bedeutung zukommt. Die funktionale Relevanz bzw. der Wirkungsmechanismus bei den Platinpräparaten in der Chemotherapie liegt insbesondere in der Hemmung der Tumorzellteilung. Basierend auf diesen
Erkenntnissen werden seit 1962 platin-basierte Medikamente entwickelt. In diesem Zusammenhang ist zunächst Cisplatin zu nennen, das seit 1977 zu den weltweit meistverwendeten zellteilungshemmenden Medikamenten zählt. Es wird bei der Krebsbehandlung in der Chemotherapie (auch bekannt als Zytostatika) eingesetzt. Eine Weiterentwicklung des Platinkomplexes ist Carboplatin (1986) bzw. Oxaliplatin, die intravenös verabreicht werden.

Der cis-Platinkomplex $\left[\mathrm{Pt}\left(\mathrm{NH}_{3}\right)_{2} \mathrm{Cl}_{2}\right]$ und seine Verwandten gehören $\mathrm{zu}$ den wirksamsten Chemotherapeutika. Ihre zytostatische Wirkung basiert auf Quervernetzungen der DNA durch die Bindung des Platinatoms an zwei Nukleinbasen. Ihr Haupteinsatzgebiet sind Brustkrebs, Gebärmutter- und Eierstockkrebs, Hodenkrebs sowie Tumore der Halsregion. Nach der Injektion ist der Großteil der Cisplatinmoleküle an Blutplasmaproteine gebunden. Der ungebundene Anteil wird durch das Blut transportiert. Erreicht ein Cisplatinmolekül eine Zelle - im Idealfall eine Tumorzelle - entfaltet es seine zytostatische Wirkung. Es gelangt durch die Zellwände verschiedener Organe und des Tumors und bildet einen reaktiven Platinkomplex. Dieser erhält seine Wirksamkeit durch Bindung an die DNA, in deren Konsequenz die DNA-Replikation gestört ist und somit das Zellwachstum nachhaltig gehemmt wird. Das neuere Platin-Analogon Oxaliplatin wird zurzeit bei Darmkrebs eingesetzt. Inzwischen gibt es bereits erste platin-basierte Krebsmedikamente mit oraler Darreichungsform, wie etwa das sich derzeit noch in der Testphase befindliche Satraplatin, bei dem weit geringere Mengen Platin benötigt werden und das zur Behandlung von Prostatakrebs entwickelt wird $[10,11]$. 
Die Zubereitung der Zytostatika findet entweder in Krankenhaus- oder Offizinapotheken statt, die als Dienstleister zentral für das Krankenhaus oder onkologische Praxen arbeiten. Die Zytostatikalösungen werden patientenindividuell dosiert und rezepturmäßig aus handelsüblichen Fertigarzneimitteln hergestellt [11]. Beim Umgang mit Zytostatikalösungen muss ein hoher Sicherheitsstandard sowohl für das herstellende als auch für das applizierende Personal eingehalten werden. Gewährleistet wird dies durch Fachausbildungen des Personals, die Einhaltung von Qualitätssicherungsstandards und relevante Guidelines [12].

Auch mengenmäßig spielen platinhaltige Zytostatika eine zunehmend bedeutende Rolle. So werden etwa bei einer einzigen Chemotherapieinfusion, wie sie zur Behandlung von Brustkrebspatientinnen eingesetzt wird, in Abhängigkeit der Größe und des Gewichtes einer Patientin zwischen 58,5 und 142,35 mg reines Platin pro Sitzung verabreicht [b]. Dies führt - bezogen auf ein Brustzentrum einer mittleren deutschen Stadt mit ca. 250 Patientinnen - zu einem Bedarf von insgesamt 200 g pro Jahr. Bei derzeit 238 zertifizierten Brustzentren in Deutschland ergibt sich dabei eine Verbrauchsmenge von ca. 50 kg/Jahr. Erweitert man die Betrachtungsebene auf alle Krebserkrankungen, werden bereits heute 6,9 t Platin pro Jahr weltweit zur Herstellung entsprechender Medikamente bzw. in der Biomedizin (z.B. für Herzschrittmacher) benötigt [4]. Dies entspricht rund 3\% der Weltjahresproduktion. Da Krebserkrankungen zu den häufigsten Todesursachen in den Industrieländern gehören und in den letzten Jahren die Krebsrate in Deutschland um 30\% gestiegen ist (jährlich erkranken 450.000 Menschen neu an Krebs), wird die Nachfragemenge nach antineoplastischen Arzneimitteln und damit auch nach platin-basierten Krebsmedikamenten in den nächsten Jahre weiter steigen. Dies gilt in umso höherem Maß, wenn derartige Therapieformen auch in Schwellenländern vermehrt eingesetzt werden. Potentielle Substitute bzw. Alternativ-Präparate in der Krebsforschung sind vor allem Paclitaxel bzw. Docetaxel, die grundsätzlich aus Extrakten der Eibe hergestellt werden bzw. mittlerweile auch durch Fermentation und Partialsynthese, und nachgewiesene zytotoxische Eigenschaften besitzen. Im Jahr 2008 betrug der weltweite Umsatz dieser Präparate bereits 4,1 Mrd. Euro [13]. Bislang werden diese Wirkstoffe allerdings oft nur als CoMedikation eingesetzt und ersetzen so platinhaltige Zytostatika nur partiell.

\section{Risiken der Dissipation platinhaltiger Katalysatoren und Zytostatika}

Aus ökologischer Sicht ist insbesondere die Dissipation von nanoskopischen Funktionsmaterialien, wie sie in platinhaltigen Stäuben oder Verbindungen $\mathrm{zu}$ finden sind, kritisch zu sehen. Auf diese Weise kommt es nicht nur $\mathrm{zu}$ einem unkontrollierten Eintrag potentieller Schadstoffe in die Biosphäre, es entstehen auch Kreislaufverluste, die zu einer zusätzlichen Nachfrage nach Primärplatin führen. Die Art und Menge des dissipierten Platins ist dabei unmittelbar von der funktionalen Verwendung abhängig [14].

So dissipieren bei der Verwendung von Platin in Autoabgaskatalysatoren - bezogen auf die durchschnittliche Lebensdauer, die bei etwa ca. $80.000 \mathrm{~km}$ liegt ca. 10\% des eingebrachten Schwermetalls als Feinstaubpartikel [15]. Dies führt zu einer Gesamtemission von Platinpartikeln aus Autoabgaskatalysatoren von ca. 5 - 20 t Platin pro Jahr in Europa, Japan und Nordamerika [16]. Auf der anderen Seite kann das nicht dissipierte Platin der Katalysatoren nach dem heutigen Stand der Technik weitgehend rezycliert werden.

Ganz anders stellt sich die Situation bei der Dissipation platinhaltiger Zytostatika dar. Hier liegen wasserlösliche Platinsalze vor, die mit dem Urin der Patienten ausgeschieden werden. Die höchste Platinkonzentration, die in Studien nachgewiesen wurde (zwei Stunden nach Applikation), beträgt $3.580 \mathrm{ng} / \mathrm{l}$ [17]. Dabei ist $\mathrm{zu}$ berücksichtigen, dass die Ausscheidung platinhaltiger Rückstände im Urin je nach verabreichter Dosis und Therapieart innerhalb der ersten 24 Stunden nach der Applikation lediglich 10 - 50\% beträgt [18]. Die exakte Feststellung von Arzneimittelrückständen aus pharmakologischen Platinprodukten in der Umwelt erweist sich aufgrund der langfristigen Verweildauer der Platinverbindungen im menschlichen Körper und der Tatsache, dass der Ort der Verabreichung von Zytostatika in der heutigen Zeit nicht mehr ausschließlich auf das Krankenhaus beschränkt ist, als problematisch (80\% der Substanzen werden vom Patienten $\mathrm{zu}$ Hause ausgeschieden) [19].

Kliniken, die ihre Patienten während der Krebsbehandlung mit einer Chemotherapie versorgen, unterliegen strengen Verfahren der Abwassersammlung und -reinigung bei Zytostatika. In diesem Zusammenhang sind hier die EU Gesetzgebung zur Vermeidung von Emissionen von kanzerogenen/mutagenen Stoffen im Abwassersystem (Council Directive 80/68/EWG und 76/464/EWG), die Gefahrstoffverordnung sowie die technischen Regeln für Gefahrstoffe und Zytostatikarichtlinien der Länder zu nennen [20].

Die platinhaltigen Rückstände, die erst zu Hause ausgeschieden werden, gelangen in fast allen Fällen in die kommunale Abwasserentsorgung. Dieser Effekt wird durch die Zunahme ambulanter Zytostatikainfusionen noch verstärkt. Zusätzliche Brisanz erhält die Anreicherung platinhaltiger Zytostatika in der Umwelt mit der Zulassung von oral zu verbreichenden Medikamenten 
(wie etwa das Satraplatin), die dann in die häusliche Toilette eingebracht werden würden.

Bezüglich der Akkumulation von Platinverbindungen in der Biosphäre ist festzuhalten, dass sich derzeit die meisten Studien auf Emission aus dem Bereich der Autoabgaskatalysatoren beziehen. Dabei kann gezeigt werden, dass Straßenstaub- und Erdproben, aber auch Wasserproben von Flüssen oder Seen, die an viel befahrenen Straßen liegen, eine signifikant erhöhte Platinkonzentration aufweisen [21]. Auch zeigen bereits Studien eine Akkumulation platinhaltiger Arzneimittelrückstände in Abwässern [22]. So wurde im Jahr 1996 von Kümmerer et. al. [17, 23] eine Gesamtemission in Deutschland von 14,2 kg Platin aus der Freisetzung platinhaltiger Medikamente aus Krankenhäusern über die Abwassersysteme in die Kläranlagen und nachgeschalteten Boden-Wasser-Systeme errechnet. Vergleicht man diese Freisetzung mit der aus den Fahrzeugkatalysatoren stammenden Menge mit 187,2 kg Platin, ist sie zwar geringer, aber dennoch beachtenswert.

Bezüglich der Risiken einer zunehmenden Platindissipation in die Biosphäre besteht weiterhin Forschungsbedarf. Dennoch deuten neuere Erkenntnisse darauf hin, dass platinhaltige Substanzen schlecht abbaubar sind und ein hohes Bioakkumulationspotential aufweisen. Ferner ist Platin - wie alle Schwermetalle - ab einer bestimmten Konzentration im Boden toxisch für Mikroorganismen, Bodentiere und Pflanzen. So belegen Studien im zoologisch-hydrologischen Fachbereich, dass Platinpartikel, die aus den Autoabgaskatalysatoren über das Regenwasser in das aquatische Ökosystem gelangen, Veränderungen bei Tieren, wie beispielsweise Zebrafischen und Posthornschnecken hervorrufen. So konnte u.a. bei den Embryonen ein Gewichtsverlust, Entwicklungsstörungen oder die Verlangsamung der Herzfrequenz beobachtet werden [24]. Über die Biosphäre kann es auch zu einer Anreicherung in der Nahrungskette kommen, so dass auch Menschen gesundheitlich beeinträchtigt werden können [25].

Die gesundheitlichen Risiken der Platindissipation für den Menschen hängen von der Art der Verbindungen und der Konzentration ab. Das Gefährdungspotential der aus der Dissipation von platinhaltigen Autokatalysatoren stammenden Feinpartikel ist noch nicht eindeutig belegt. Anders sieht es bei der Emission von Palladium aus, das vor allem in Katalysatoren von Dieselmotoren verwendet wird. So ist für Palladiumstäube eine (öko-) toxikologische Wirkung nachgewiesen [21].

In Bezug auf Zytostatika liegen die gesundheitlichen Risiken für den Menschen im Umgang bei der Zubereitung, bei der Applikation und in der Ausscheidung durch den Urin ins Abwasser. Bei der Zubereitung können allergische Reaktionen der Haut und der Schleimhäute auftreten [15]. Eine kanzerogene
Wirkung besteht bei der Zytostatikaapplikation durch Cisplatin, d.h. die Nachweise bei Menschen sind zwar begrenzt, die Nachweise bei Tieren jedoch ausreichend [23].

Besonders problematisch erscheint die Emission von Platinsalzen, die durch die Ausscheidung von Zytostatika über den Urin frei gesetzt werden. Zukunftsorientierte Sanitärkonzepte und Weiterentwicklungen bestehender Abwasserbeseitigungssysteme sind in Zukunft erforderlich, die eine Verminderung von Pharmakawirkstoffen im Abwasser über die Trennung von Stoffströmen bewirken [26]. Optionen zur Reduktion der beschriebenen Risiken für die Biosphäre liegen einerseits in einer Verbesserung der Effizienz der Trennung platinhaltiger Abwässer, etwa durch die Entwicklung zukunftsweisender Sanitärkonzepte bzw. die Weiterentwicklungen bestehender Abwasserbeseitigungssysteme. So zeigt eine aktuelle Studie die positive Wirkung von UV-Licht auf die Zersetzung von Platinverbindungen aus Zytostatika [18].

\section{Reduktion ökonomischer und ökologischer Risiken durch Umsetzung einer Kreislaufwirtschaft}

Ein prinzipielles Konzept zur Reduktion ökonomischer und ökologischer Risiken kritischer Stoffe liegt in der Schaffung von Stoffkreisläufen bzw. Kreislaufwirtschaftssystemen (Closed Loop Supply Chain Management) $[27,28]$. Stoffkreisläufe führen zu einer Erhöhung der Ressourceneffizienz und damit zu einer Verlängerung der Reichweite bei gleichzeitiger Reduktion des Stoffeintrages in die Biosphäre. Bei der Umsetzung von Kreislaufwirtschaftssystemen können grundsätzlich drei Handlungsalternativen unterschieden werden. Bei Reuse- und Remanufacturing-Strategien werden Produkte bzw. Komponenten nach einer entsprechenden Aufarbeitung wiederverwendet. Beim Recycling wird dagegen der in Alt-Produkten enthaltene Grundstoff etwa durch Einschmelzen mit anschließender Elektrolyse wiedergewonnen. Dieser so gewonnene Sekundärrohstoff kann dann als Substitut des Primärrohstoffes zu Beginn der Wertschöpfungskette eingesetzt werden. Aus ökologischer Sicht ist die Verwendung von Sekundärrohstoffen wie Sekundärplatin meist vorteilhaft. So müssen bei der Gewinnung von $1 \mathrm{~kg}$ Primärplatin bis zu $150 \mathrm{t}$ an Gestein aus bis zu 1000 Metern Tiefe gefördert und anschließend chemisch aufbereitet werden. Hierbei entstehen fast $400 \mathrm{t}$ zu entsorgendes Abraummaterial sowie Schlacke. Im Gegensatz dazu benötigt die Rückgewinnung von Platin aus Autokatalysatoren nur $2 \mathrm{t}$ an Umwandlungsmaterial oder $500 \mathrm{~kg}$ Keramiksubstrat und der Prozess ist technologisch einfacher beherrschbar $[7,8]$.

Untersucht man die bestehenden Rückführungssysteme für Platin, so finden sich diese insbesondere für Produkte der Schmuckindustrie und der Autoabgaskatalysatoren. 
Derzeit werden in Deutschland 52\% der Autokatalysatoren und in den Vereinigten Staaten 35\% der Autoabgaskatalysatoren rezycliert. Die Gesamtmenge von Sekundärplatin liegt weltweit bei ca. 20 t/Jahr und damit bei rund $10 \%$ der Gesamtnachfrage. Gründe hierfür liegen in der niedrigen Produktkonzentration, dem Stand der Rückgewinnungstechnologien sowie organisatorischen Hemmnissen bei der Etablierung entsprechender Kreislaufwirtschaftssysteme [5]. Problemtisch in Bezug auf eine Erhöhung der Rückführungsquote ist darüber hinaus das Phänomen der Dissipation.

Das Recycling von Wirkstoffen aus Medikamenten kann unter dem Begriff "Green and Sustainable Pharmacy" [29] eingeordnet werden. Nach Aussagen eines britischen Chemieunternehmens, das sich auf das Recycling von Katalysatoren, Edelmetallen und Feinchemikalien spezialisiert hat, könnte Platin grundsätzlich aus dem Urin rezycliert werden. Die Wirtschaftlichkeit entsprechender Recyclingverfahren hängt jedoch von der Konzentration im Urin der Patienten ab, diese wiederum vom applizierten Platinkomplex. Die höchsten Konzentrationen ergeben sich bei Carboplatin, gefolgt von Oxali- und Cisplatin. Die gemessene Maximalkonzentration (3.580 ng/l zwei Stunden nach Applikation) bzw. die Durchschnittskonzentration pro Tag (10 - 660 ng/l) steht jedoch einem ökonomischen Recycling entgegen, auch wenn sie - verglichen mit den Normalwerten (0,5 ng/l - 4,1 ng/l) bzw. dem entsprechenden Referenzwert des Umweltbundesamtes bei Erwachsenen (10 ng/l) - deutlich erhöht sind [17,21,30]. Im Gegensatz dazu ist das Recycling von Platin aus abgelaufenen Medikamenten möglich [c].

Unabhängig davon wäre es aus kreislauforientierter Sicht wünschenswert, eine Trennung der Abwasserströme vornehmen zu können. Grundsätzlich können die Teilströme Grauwasser (Wasser, das zum Waschen von Körper und Kleidung genutzt wird), Gelbwasser (Urin), Braunwasser (Wasser vermischt mit festen Exkrementen) und Schwarzwasser (gesamtes Abwasser, das in die Kanalisation fließt) getrennt gesammelt und aufbereitet werden. Dies schafft die Voraussetzung, dass jeder Abwasserstrom weiterverwendet werden kann. Gelbwasser wird für Düngemittel verwendet, aus Braunwasser lässt sich Energie gewinnen und Grauwasser kann zum Gießen und zum erneuten Waschen verwendet werden. Andererseits wird auf dem Gebiet der Forschung über Abwasserrecycling seit ca. 10 Jahren gearbeitet. Neuere Ergebnisse zeigen, dass beispielsweise die Rückgewinnung von Phosphaten im Rahmen neuartiger Sanitärsysteme (NASS) möglich ist [31].

\section{Diskussion und Zusammenfassung}

Die zukünftige wirtschaftliche Entwicklung moderner Industrieländer hängt in hohem Maße von der
Verfügbarkeit intelligenter Werkstoffe und Materialien als Basis innovativer Produkte bzw. entsprechender funktionaler Einheiten ab - etwa im Bereich der Luft- und Raumfahrt, des Anlagenbaus, der Automobilindustrie oder der Prozess- und Pharmaindustrie. Grundlage entsprechender Produkte oder Funktionsgruppen sind oftmals knappe Rohstoffe bzw. Ressourcen, deren Erschließung und Verarbeitung mit zunehmend steigenden Kosten und ökologischen Risiken verbunden sind. Nach der Nutzungsphase dissipieren die eingesetzten Wertstoffe oftmals und begrenzen so nicht nur die Produktion innovativer Produkte und damit die gesamtgesellschaftliche Wohlfahrt, sondern gefährden darüber hinaus die Biosphäre durch den Eintrag entsprechender Schadstoffe. Vor diesem Hintergrund muss die Analyse und Bewertung der Kritikalität einer Ressource den gesamten Lebenszyklus von der Förderung des jeweiligen Rohstoffes, der Produktions- und Nutzungsphase bis hin zur Nachnutzungsphase und Dissipation umfassen. Auf allen Stufen sind gleichermaßen ökonomische und ökologische Kriterien zu berücksichtigen. Ein zentraler Ansatzpunkt zur Reduktion der ökonomischen und ökologischen Risiken besteht in einer Umsetzung von Kreislaufwirtschaftssystemen (Closed Loop Supply Chains). Da insbesondere die Dissipations- und Rückführungsprozesse stark von der jeweiligen funktionalen Verwendung der betrachteten Ressource abhängen, muss eine Kritikalitätsbewertung immer auch funktionale Kriterien umfassen. Im vorliegenden Artikel wird dieses Phänomen stellvertretend für die Ressource Platin als Wirkstoff von Zytostatika bzw. aktives Funktionsmaterial von Autoabgaskatalysatoren diskutiert. Tabelle 1 fasst die wesentlichen Ergebnisse des Kritikalitätsassessments zusammen.

Dabei zeigt sich, dass die ressourcenspezifischen Risiken der Ressource Platin im Wesentlichen in der geographischen Verteilung der Quellen, der energieintensiven Exploration sowie der Tatsache, dass die Ressource Platin oft nur als Kuppelprodukt anderer Metalle abgebaut wird, liegen. Bezüglich der Reichweite ist anzumerken, dass diese einerseits von der ökonomischen Dynamik etwa im Bereich der Entwicklung von Brennstoffzellen und Medikamenten sowie technischer Innovationen abhängt, andererseits von den identifizierten Reserven sowie den hypothetisch vorhandenen Ressourcen in der Erdkruste .

Aufgrund des technischen Fortschritts und der Verbreitung moderner Pharmazeutika in Schwellenländern sowie der alternden Bevölkerung in Industrieländern erscheint die zytotoxische Eigenschaft von Platin besonders bemerkenswert. Haupteinsatzgebiete platinhaltiger Zytostatika sind Brustkrebs, Gebärmutter- und Eierstockkrebs, Hodenkrebs sowie 


\begin{tabular}{|c|c|c|}
\hline Ressourcenspezifische Kriterien & \multicolumn{2}{|c|}{ Autoabgaskatalysatoren/Zytostatika } \\
\hline Aktuelle Nachfrage & \multicolumn{2}{|c|}{$200-250$ t/a (Tendenz steigend)* } \\
\hline Aktuelles Fördervolumen & \multicolumn{2}{|c|}{$200 t / a^{*}$} \\
\hline Stat. Reichweite & \multicolumn{2}{|c|}{ ca. 100 Jahre } \\
\hline Geostrateg. Verteilung & \multicolumn{2}{|c|}{77 \% der Abbaugebiete in einer Region in Südafrika* } \\
\hline Ökologische Risiken & \multicolumn{2}{|c|}{ die Primärgewinnung ist äußerst energieintensiv } \\
\hline Funktionsabhängige Kriterien & Autoabgaskatalysatoren & Zytostatika \\
\hline Substitutionspotential & bei Dieselmotoren durch Palladium & partiell durch semisynthetische Wirkstoffe der Eibe \\
\hline Recyclingpotential & $50-90 \% * *$ & $\begin{array}{l}\text { setzt Entwicklung zukunftsorientierter Sanitär- } \\
\text { und Entsorgungskonzepte voraus }\end{array}$ \\
\hline Dissipation & $\begin{array}{l}\text { Platin als Feinstaubpartikel } \\
187,2 \mathrm{~kg} / \mathrm{a}^{* * *} \text { für Deutschland }\end{array}$ & $\begin{array}{c}\text { Gelöste Platinsalze } \\
20-3.580 \mathrm{ng} / \mathrm{l} \text { Platin im Urin/Patient nachgewiesen***; } \\
14,2 \mathrm{~kg} / \mathrm{a} \text { Gesamtemission aus Krankenhäusern (BRD)**** }\end{array}$ \\
\hline Bioaktivität & $\begin{array}{c}\text { Tiere }+ \\
\text { Menschen }(+)^{* * * * *}\end{array}$ & $\begin{array}{l}\text { wahrscheinlich }++ \\
\text { (Forschungsbedarf) }\end{array}$ \\
\hline Toxizität & akut wenig toxisch ${ }^{* * * * * *}$ & akut toxisch ${ }^{* * * * *}$ \\
\hline
\end{tabular}

Tumore der Halsregion. Der Erfolg entsprechender Medikamente führt dazu, dass bereits heute 6,9 t oder $3 \%$ der Platinweltjahresproduktion in der (bio-)medizinischen Branche eingesetzt werden.

Betrachtet man diese Entwicklung im Kontext von Zukunftsszenarien von anderen funktionalen Verwendungen der Ressource Platin, wie etwa der Brennstoffzelle, für die im Jahr 2030 ein Bedarf von bis zu 334 t/a prognostiziert wird, erkennt man das Konfliktpotential über die gesellschaftlich präferierte Nutzung.

Ein prinzipielles Problem der Verwendung platinhaltiger Produkte liegt im Phänomen der Dissipation. Diese hängt allerdings, wie das Recyclingpotential, stark von der jeweiligen funktionalen Verwendung ab. So werden allein durch die Verteilung von Platinpartikeln von Autokatalysatoren ca. 5 - 20 t Platin pro Jahr in Europa, Japan und Nordamerika in der Nutzungsphase in so kleinen Konzentrationen verteilt, dass sie nicht mehr zurückgewonnen werden können. Andererseits wird bereits heute $50 \%$ des in mobilen Katalysatoren eingesetzten Platins rezycliert. Die emittierten Platinpartikel stellen dabei aus heutiger Sicht nur ein begrenztes Gefährdungspotential dar. Demgegenüber beträgt die Dissipation platinhaltiger Medikamente nur $4-7$ t pro Jahr, die emittierten Substanzen gelten aber als ökologisch kritischer und können derzeit noch nicht rezycliert werden.

\section{Handlungsempfehlungen und Forschungsperspektiven}

Die vorliegende Studie hat gezeigt, dass die Bewertung der Kritikalität einer Ressource alle Wertschöpfungsphasen umfassen muss und insbesondere auch von der jeweiligen funktionalen Verwendung abhängt. Aus ökologischer Sicht erscheint neben der Förderung der Primärressource insbesondere die Dissipation kritisch.

Vor diesem Hintergrund besteht erheblicher Forschungsbedarf hinsichtlich der Umsetzung eines funktionierenden Kreislaufwirtschaftssystems für platinhaltige Produkte. Dies bezieht sich sowohl auf organisatorische Fragen (Ausgestaltung von Rückführungssystemen von Alt-Produkten) als auch auf technologische Entwicklungen (Verfahren zum Recycling von platinhaltigen Abwässern). Eine Implementierung effizienter Kreislaufwirtschaftssysteme führt durch eine Erhöhung der Ressourceneffizienz bzw. durch eine Reduktion der dissipativ freigesetzten Partikel gleichermaßen zu einer Reduktion der ökonomischen als auch der ökologischen Risiken [32].

Angesichts der steigenden Nachfrage nach Platin wird auch bei einer Entwicklung effizienter Rückführungssysteme ein Ressourcenkonflikt bezüglich der funktionalen Verwendung bestehen bleiben. Vor diesem Hintergrund ist die vorgestellte Kritikalitätsbewertung um ethische und soziale Kriterien $\mathrm{zu}$ erweitern. Gleichermaßen müssen auch zukünftige positive ökologische Effekte potentiell funktionaler Verwendungen (z.B. Reduktion von $\mathrm{CO}_{2}$ durch den Einsatz von Brennstoffzellen) entsprechend berücksichtigt werden.

\section{Acknowledgement}

Die Autoren danken der G+B Schwyzer Stiftung, Zürich/Schweiz für die finanzielle Unterstützung.

\section{Endnotes}

aDie Europäische Kommission versteht unter knappen Materialien solche, deren Zugangsrisiken hoch sind, z.B. Risiken der Verfügbarkeit oder hohe ökologische Risiken und darüber hinaus von großer ökonomischer Bedeutung 
sind [7]. Unter kritischen Metallen werden seltene Metalle verstanden, die zur Gewährleistung zentraler Funktionen für eine moderne Gesellschaft als existentiell angesehene Produkte unabdingbar erscheinen (also von großer ökonomischer Bedeutung sind) bzw. deren Substitution mit hohen ökonomischen oder ökologischen Kosten bzw. Risiken verbunden sind. ${ }^{b}$ Nach Auskunft einer Krankenhausapotheke.

"Nach Aussagen der Technologieabteilung von „Johnson Matthey".

Received: 14 February 2011 Accepted: 13 July 2011

Published: 13 July 2011

\section{References}

1. U.S. Department of Energy (Hrsg.): Critical Materials Strategy. [http://www. energy.gov/news/documents/criticalmaterialsstrategy.pdf]

2. European Commission (Hrsg.): Critical raw materials for the EU. [http:// ec.europe.eu/enterprise/policies/raw-materials/documents/index-en.htm], 2010

3. Pero-Sanz Elorz JA, Verdeja-González JI, Sancho-Martínez JP, Vilela N: Melting and sintering platinum in the $18^{\text {th }}$ century: The secret of the Spanish Journal of Minerals, Metals and Materials Society 1999, 51(10):9-13.

4] Johnson Matthey: Platinum 2010. [http://www.platinum.matthey.com/ uploaded_files/Pt_2010/german_press_release_2010.pdf]

5. Hagelüken C et. al.: Stoffströme der Platingruppenmetalle, Systemanalyse und Maßnahmen für eine nach haltige Optimierung der Stoffströme der Platingruppenmetalle. GDMB Medienverlag: 2005

6. Angerer G, Erdmann L, Marscheider-Weidemann F et al.: Rohstoffe für Zukunftstechnologien. Einfluss des branchenspezifischen Rohstoffbedarfs in rohstoffintensiven Zukunftstechnologien auf die zukünftige Rohstoffnachfrage. Karlsruhe: Fraunhofer IRB-Verlag; 2009.

7. Hagelüken C: Autoabgaskatalysatoren: Grundlagen, Herstellung, Entwicklung, Recycling, Ökologie. Renningen-Malmsheim: Expert Verlag; 2011.

8. Hagelüken C: Securing the supply for tomorrow: precious metals recovery from automotive catalysts. METALL 2001, 55:104-111.

9. Helmers E: Bitte wenden Sie jetzt, Das Auto der Zukunft. Weinheim: Wiley-VCH Verlag; 2009.

10. Ott I, Gust R: Medizinische Chemie der Platinkomplexe: Besonderheiten anorganischer Zytostatika. Pharmazie in unserer Zeit 2006, 35(2):124-133.

11. Warnke U: Patientenindividuelle Zytostatikazubereitung. Platinkomplexe in der (Krankenhaus-)Apotheke. Pharmazie in unserer Zeit 2006, 35(2):110-116.

12. Berufsgenossenschaft für Gesundheitsdienst und Wohlfahrtspflege (BGW): Zytostatika im Gesundheitsdienst. Informationen zur sicheren Handhabung von Zytostatika. [http://www.bgw-online.de/internet/generator/Inhalt/ Onlinelnhalt/Medientypen/bgw_20themen/M620_Zytostatika_im_Gesu ndheitsdienst, property=pdfDownload.pdf].

13. Schäfer B: Hoffnung gegen Krebs. Taxol ${ }^{\circledR}$. Chemie in unserer Zeit 2011, 45:32-46.

14. Reller A: Chemie im Nanobereich - Vielversprechendes oder unwägbares Neuland. Chemie in unserer Zeit 2005, 39:67-71.

15. Bleich S: Ökologisches Stoffgebiet. Stuttgart: Schattauer Verlag; 2000

16. Reller A: Chemie im Kontext - Skizze einer Geographie der Ressourcen politische ökologie 2003, 86:22-25.

17. Kümmerer K: Drugs in the environment: emission of drugs, diagnostic aids and disinfectants into wastewater by hospitals in relation to other sources - a review. Chemosphere 2001, 45:957-969.

18. Wagner $\mathrm{G}$, Weber $\mathrm{S}$, Reller A: Removal of cytotoxic platinum compounds from aqueous media by photolysis in the presence of 2,4,6-trimercapto1,3,5-triazine. The Royal Society of Chemistry 2011, in Veröffentlichung.
19. Gießen H: Platin im Abwasser. [http://www.pharmazeutische-zeitung.de/ index.php?id=pharm4_24 2004]

20. Bayerisches Staatsministerium für Gesundheit, Ernährung und Verbraucherschutz: Umgang mit Zytostatika. Ein Leitfaden für die Praxis. [http://www.uni-erlangen.de/einrichtungen/arbeitssicherheit/gefahrstoffe/ umgang/zytostatika_stmgev.pdf]

21. Hoppstock K, Sures B: Platinum-Group Metals. In Elements and their compounds in the environment. 2nd edition. Edited by Merian M, Anke M, Innat M. Wiley-VCH Verlag GmbH \& Co. KGaA; 2004:1047-1086.

22. Götz K, Keil F: Medikamentenentsorgung in privaten Haushalten: Ein Faktor bei der Gewässerbelastung mit Arzneimittelwirkstoffen ZUmweltchem Ökotox 2007, 19(3):180-188.

23. Kümmerer K: European hospitals as a source of platinum in the environment in comparison with other sources. Science of the Total Environment 1999, 225(1-2):155-165.

24. Osterauer R, Haus N, Sures B, Köhler HR: Uptake of platinum by zebrafish (Danio rerio) and ramshorn snail (Marisa cornuarietis) and resulting effects on early embryogenesis. Chemosphere 2009, 77(7):975-982

25. Kümmerer K: Herkunft, Vorkommen, Verhalten und Verbleib von Arzneimitteln in der aquatischen Umwelt. In Praktische Krankenhaushygiene und Umweltschutz. Edited by Dettenkofer M, Frank U, Scherrer M, Daschner F. Berlin: Springer Medizin Verlag; 2006:285-290.

26. Püttmann W, Keil F, Oehlmann J, Schulte-Oehlmann U: Wassertechnische Strategien zur Reduzierung der Trinkwasserbelastung durch Arzneimittelwirkstoffe. Umweltwiss Schadst Forsch 2008, 20:209-226.

27. Tuma A, Lebreton B: Zur Bewertung und Umsetzung von Kreislaufwirtschaftsstrategien. ZfB-Special 2005, 3:59-75.

28. Dekker R, Fleischmann M, Inderfurth K, Wassenhove van L: Reverse Logistics - Quantitative Models for Closed-Loop Supply Chains. Berlin, Heidelberg: Springer Verlag; 2004

29. Kümmerer K, Hempel M: Green and Sustainable Pharmacy. Berlin: Springer Verlag; 2010

30. Alt F, Eschnauer HR, Mergler B, Messerschmitdt J, Tölg G: A contribution to the ecology and enology of platinum. Fresenius J Anal Chem 1997, 357:10131019. UBA-Literatur.

31. Horn von J, Sartorius C, Tettenborn F: Technologievorausschau für Phosphorrecyclingtechnologien. [http://www.phosphorrecycling.de/ attachments/041 Bericht AP6 Technologievorausschau 2 010-08-10.pdf

32. Oehme I, Weiland-Wascher A, Behrendt S, Erdmann L: Klimaschutz und Ressourceneffizienz. Herausforderungen und Marktchancen für die Informationswirtschaft und Telekommunikation. [http://www.umweltdaten. de/publikationen/fpdf-1/3432.pdf]

33. International Agency for Research on Cancer: IARC Monographs on the Evaluation of

Carcinogonic Risks to Humans. [http://monographs.iarc.fr/ENG/Classification/ index.php]

34. Daunderer M: Handbuch der Umweltgifte. Landsberg: ecomed MEDIZIN Verlag; 2006

doi:10.1186/2190-4715-23-26

Cite this article as: Thorenz A, Reller A: Discussion of risks of platinum resources based on a function orientated criticality assessment - shown by cytostatic drugs and automotive catalytic converters. Environmental Sciences Europe 2011, 23:26. 